\title{
Type 2 Diabetes Mellitus is an Independent Risk Factor for Postoperative Complications in Patients Surgically Treated for Meningioma
}

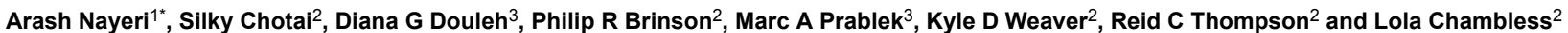

${ }^{1}$ Vanderbilt University Medical Center, 1161 21st Ave S, Rm T-4224 MCN, Nashville, TN 37232-2380, USA

2Department of Neurosurgery, Vanderbilt University Medical Center, 1161 21st Ave S, Rm T-4224 MCN, Nashville, TN 37232-2380, USA

${ }^{3}$ Vanderbilt University School of Medicine, 201 Light Hall, Nashville, TN 37232, USA

*Corresponding author: Arash Nayeri, Vanderbilt University Medical Center, 1161 21st Ave S, Rm T-4224 MCN, Nashville, TN 37232-2380, USA, Tel: 310-383-5085; Email: Arash.nayeri@vanderbilt.edu

Received date: March 22, 2016; Accepted date: April 20, 2016; Published date: April 27, 2016

Copyright: (C) 2016 Nayeri A, et al. This is an open-access article distributed under the terms of the Creative Commons Attribution License, which permits unrestricted use, distribution, and reproduction in any medium, provided the original author and source are credited.

\begin{abstract}
Objectives: Increased risk of perioperative complications in patients with type 2 diabetes mellitus (DM) has previously been noted with regard to a number of different operations. We sought to study the relative rates of postoperative complications after the surgical resection of an intracranial meningioma based on a pre-existing diagnosis of diabetes.

Methods: We conducted a retrospective cohort study on 259 patients who underwent a primary meningioma resection at our institution between 2001-2013. The medical record was reviewed to identify a pre-existing diagnosis of type $2 \mathrm{DM}$ and any postoperative complications prior to discharge. The duration of postoperative hospital stay, intensive care unit (ICU) stay, perioperative changes in Karnofsky Performance Status (KPS) scores, and any postoperative emergency department (ED) presentation within 90 days of the operation were also recorded for each patient. Multivariable logistic regression models were built to determine the impact of a history of diabetes on postoperative complications and post-discharge presentation to the ED. Multivariable linear regression models were designed to assess the predictors of lengthier hospitalization and ICU stays in addition to differential postoperative changes in KPS scores.
\end{abstract}

Results: Forty-one (16\%) patients had diagnoses of type 2 DM prior to clinical presentation. In multivariate analyses, patients with a pre-existing history of diabetes had a higher risk of postoperative complications, postoperative ED presentation, and deterioration in functional status in addition to lengthier durations of hospitalization and ICU stay $(p<0.001, p=0.008, p<0.001, p=0.007, p<0.001)$.

Conclusions: Patients with pre-existing diagnoses of type 2 DM have a significantly increased risk of immediate postoperative complications following the resection of an intracranial meningioma. Type 2 DM also predicts increased lengths of postoperative hospital stay, decreased postoperative performance status, and increased risk of postoperative ED presentation.

Keywords: Meningioma; Diabetes; Postoperative complication; KPS; ED

\begin{abstract}
Abbreviations
BMI: Body mass index; CVA: Cerebrovascular accident; DM: Diabetes mellitus; DVT: Deep venous thrombosis; ED: Emergency Department; EVD: External Ventricular Drain; GTR: Gross-total Resection; ICU: Intensive Care Unit; KPS: Karnofsky Performance Status; MI: Myocardial Infarction; MRI: Magnetic resonance imaging; OR: Odds Ratio; PE: Pulmonary embolus; UTI: Urinary tract infection; VPS: Ventriculoperitoneal Shunt; WHO: World Health Organization
\end{abstract}

\section{Highlights}

-Postoperative complications following meningioma resection are diverse in etiology.
-Type 2 diabetes predicts increased risk of complications following meningioma resection.

-Type 2 diabetes also predicts lengthier hospital stays and a decrease in performance status.

-Postoperatively, diabetics present to the ED at higher rates.

-A diagnosis of diabetes should be considered when stratifying risk in these patients.

\section{Introduction}

Intracranial meningiomas are the most frequently diagnosed primary brain tumors, accounting for roughly $1 / 3$ of all diagnoses $[1,2]$. The majority of meningiomas carry a favorable prognosis, and amongst the operative cases the risk of postoperative morbidity and mortality is fairly low [3-5]. However, a number of factors have been associated with higher risks of postoperative complications. Patient age, poor preoperative performance status, and tumor location are the 
Citation: Nayeri A, Chotai S, Douleh DG, Brinson PR, Prablek MA, et al. (2016) Type 2 Diabetes Mellitus is an Independent Risk Factor for Postoperative Complications in Patients Surgically Treated for Meningioma. J Neurol Neurophysiol 7: 368. doi: $10.4172 / 2155-9562.1000368$

Page 2 of 6

most consistently identified predictors of increased risk of complications following meningioma resection [6-10].

In recent years, there has been improved recognition of the relationship between type 2 diabetes mellitus (DM) and perioperative complications following a variety of surgical procedures [11-13]. Type 2 DM has also been associated with longer postoperative hospitalizations and increased costs to the healthcare system. In a number of different surgical patient populations, a pre-existing diagnosis of diabetes also portends worse outcomes post-discharge and predicts increased risk of readmission subsequent to the initial procedure $[14,15]$.

Of note, a pre-existing diagnosis of type $2 \mathrm{DM}$ has also been identified as an independent risk factor for certain neurological complications following craniotomy, including meningitis and CSF leaks [16,17]. However, in such studies a heterogeneous group of patients with different indications for craniotomies are often grouped together. Moreover, non-neurological complaints are often not assessed in these studies. We sought to study the role of type $2 \mathrm{DM}$ in predicting short-term postoperative complications in patients undergoing surgical resection of an intracranial meningioma. We aim to show that a pre-existing diagnosis of type $2 \mathrm{DM}$ is independently associated with an increased risk of systemic and neurological complications in addition to lengthier postoperative hospital and ICU stays, deterioration of performance status, and increased risk of postoperative ED presentation in this patient population.

\section{Methods}

\section{Patient population}

We conducted a retrospective cohort study on 259 patients who had a primary intracranial meningioma resected at our institution between 2001-2013. For patients with a history of multiple meningioma resections at our institution, only data with regard to the initial resection was used in the study. Four attending neurosurgeons with specialized training in neurosurgical oncology performed all resections.

\section{Data collection and study variables}

Records of clinical and radiographic data were retrospectively obtained from the electronic medical record and entered into a REDCap database [18]. All such entries in the medical record were reviewed from the time of initial clinical presentation to date of last chart review, 10/17/2015. Patient age, gender, pre-existing diagnoses of type $2 \mathrm{DM}$, body mass index (BMI), perioperative blood glucose levels, extent of surgical resection, tumor pathological grade, details of any radiation therapy, and perioperative Karnofsky Performance Status (KPS) scores were recorded for each patient. Additionally, any of the pre-determined components of a postoperative complication, length of postoperative hospital and ICU stay, and any postoperative emergency department (ED) presentation within 90 days of the operation were also recorded for each patient.

A duration of follow-up variable was defined as the time between the operation and the last clinic visit with the attending neurosurgeon. Patient age was defined as age at time of operation. A pre-existing diagnosis of type $2 \mathrm{DM}$ variable was created based on a clinical history of type $2 \mathrm{DM}$ at least 6 months prior to the radiographic diagnosis of an intracranial meningioma. Patients with a history of type $1 \mathrm{DM}$ were excluded from the study.
Patients with new diagnoses of type $2 \mathrm{DM}$ subsequent to clinical presentation and surgical resection of the meningioma were not designated as diabetics in this study. BMI was calculated from the preoperative patient assessment. A preoperative blood glucose level variable was defined based on the latest available fasting blood glucose prior to the date of surgery. A postoperative blood glucose level variable was defined as the patient's mean blood glucose between the end of the operation and time of discharge. All blood glucose levels are reported in $\mathrm{mg} / \mathrm{dL}$.

Extent of surgical resection was classified as gross-total resection (GTR) or incomplete resection based on the interpretation of the immediate postoperative magnetic resonance imaging (MRI) scan by the neuroradiologist. Tumor grade was assigned as WHO Grade I, II, or III based on the neuropathologist's assessment of the tumor subsequent to the resection. KPS scores were assigned based on clinical assessment, both preoperatively at time of presentation and postoperatively at time of first postoperative clinic visit.

A postoperative complication variable was defined as the occurrence of any of the following between the time of the operation and discharge: urinary tract infection (UTI), deep venous thrombosis (DVT), pulmonary embolus (PE), wound infection, pneumonia, transfusion requirement, myocardial infarction (MI), cerebrovascular accident (CVA), external ventricular drain (EVD) or ventriculoperitoneal shunt (VPS) placement, seizure, and death. A length of hospital stay variable was defined as the number of days between the operation and discharge from the hospital. A length of ICU stay variable was defined as the number of days the patient spent in the intensive care unit (ICU) following the operative resection.

A postoperative ED presentation variable was created based on any patient presentation to an ED within 90 days of the operation. The medical record for each patient was reviewed for any entry that noted a relevant ED visit at an outside hospital or to our institution. No distinction was made between ED presentation to our institution and others. Patients with duration of follow-up of less than 90 days were excluded from the analysis of postoperative ED presentations.

\section{Statistical analysis}

All information was de-identified prior to statistical analysis in Microsoft Excel (Redmond, WA, USA) and IBM SPSS Statistics (Armonk, NY, USA) [19,20]. Mean, standard deviation, and median were computed for continuous variables; frequency and percentage were computed for categorical variables. Descriptive statistics were performed using Fisher's exact test for categorical variables and student's $t$ test for continuous variables. Multivariable logistic regression models were built to determine the impact of a history of diabetes on postoperative complications and post-discharge presentation to the ED.

Multiple linear regression models were designed to assess the predictors of lengthier hospitalization and ICU stays in addition to differential postoperative changes in KPS scores. Variables with a $\mathrm{p}$ value of more than 0.05 were removed from the linear models in a step-wise fashion. The independent variables including age, gender, BMI, preoperative glucose, postoperative glucose, preoperative KPS, and extent of surgical resection were included in the models. For all analysis, a p-value of less than or equal to 0.05 was considered statistically significant. 
Citation: Nayeri A, Chotai S, Douleh DG, Brinson PR, Prablek MA, et al. (2016) Type 2 Diabetes Mellitus is an Independent Risk Factor for Postoperative Complications in Patients Surgically Treated for Meningioma. J Neurol Neurophysiol 7: 368. doi: $10.4172 / 2155-9562.1000368$

Page 3 of 6

\section{Results}

A total of 259 patients met the inclusion criteria and were evaluated in this study. The mean age at time of operative resection was $55.1 \pm$ 14.9 years. The patient population included 185 (71\%) females and 74 (29\%) males. Mean BMI at time of surgical intervention was $30.1 \pm 8.7$. GTR of the meningioma was accomplished in 207 (80\%) patients. The mean preoperative KPS score was $76.7 \pm 11.3$. Mean preoperative blood glucose in all patients was $116.5 \pm 47.7 \mathrm{mg} / \mathrm{dL}$. Postoperatively, mean blood glucose had increased to $165.5 \pm 36.8 \mathrm{mg} / \mathrm{dL}(\mathrm{p}<0.001)$. Mean duration of postoperative follow-up was $41.9 \pm 32.0$ months.

Forty-one (16\%) patients had pre-existing diagnoses of type $2 \mathrm{DM}$ prior to clinical presentation and $218(84 \%)$ were non-diabetics. Statistically significant differences were found between diabetics and non-diabetics with regard to age at time of operation, gender, mean BMI, in addition to mean preoperative and postoperative blood glucose levels. No significant difference was noted between the two groups with regard to extent of surgical resection, mean preoperative KPS, or duration of postoperative clinical follow-up. These findings are summarized in Table 1.

\begin{tabular}{|l|c|c|c|c|}
\hline & All Patients & Diabetics & $\begin{array}{c}\text { Non- } \\
\text { Diabetics }\end{array}$ & P Value \\
\hline \# of Patients & 259 & 41 & 218 & N/A \\
\hline Mean age (years) & 55.1 & 63.2 & 53.5 & $<0.001$ \\
\hline Gender (\% female) & 71 & 54 & 75 & 0.008 \\
\hline BMl (mean) & 30.1 & 33.7 & 29.4 & 0.003 \\
\hline $\begin{array}{l}\text { Extent of surgical } \\
\text { resection (\% GTR) }\end{array}$ & 80 & 90 & 78 & 0.089 \\
\hline $\begin{array}{l}\text { Pre-operative KPS } \\
\text { (mean) blood }\end{array}$ & 116.5 & 151.5 & 109.8 & $<0.001$ \\
\hline $\begin{array}{l}\text { Pre-operative } \\
\text { glucose (mean) }\end{array}$ & 165.5 & 195.5 & 159.7 & $<0.001$ \\
\hline $\begin{array}{l}\text { Post-operative blood } \\
\text { glucose (mean) }\end{array}$ & 41.9 & 35.7 & 43.1 & 0.171 \\
\hline $\begin{array}{l}\text { Mean postoperative } \\
\text { follow-up (months) }\end{array}$ & 16.4 & 76.9 & 0.417 \\
\hline
\end{tabular}

Table 1: Basic demographic and clinical features. Patients with type 2 DM were on average older, less female predominant, and had a larger BMI in addition to higher preoperative and postoperative blood glucose measurements.

The patients' charts were reviewed for a number of neurological and non-neurological complications subsequent to the operative resection. Overall, 49 total complications were identified in 30 patients. Thirteen (32\%) patients with diabetes and 17 (8\%) non-diabetics suffered at least one complication postoperatively. The relative risk of postoperative complications in diabetics was found to be statistically significant $(\mathrm{p}<0.001)$. A summary of the postoperative complications in provided in Table 2.

A multiple variable logistic regression analysis was conducted to assess for independent predictors of postoperative complications by controlling for age, gender, BMI, preoperative and postoperative blood glucose levels, preoperative KPS scores, extent of resection, and WHO grade. Patients with a pre-existing history of type $2 \mathrm{DM}(\mathrm{OR}=4.8,95 \%$
$\mathrm{CI}=2.1$ to $11.3, \mathrm{p}<0.001)$, male patients $(\mathrm{OR}=2.7,95 \% \mathrm{CI}=1.1$ to 6.6 , $\mathrm{p}=0.036)$, and patients with incomplete resections ( $\mathrm{OR}=3.7,95 \%$ $\mathrm{CI}=1.1$ to $6.7, \mathrm{p}=0.011$ ) had higher odds of suffering from at least one postoperative complication.

\begin{tabular}{|l|l|l|l|l|}
\hline & $\begin{array}{l}\text { All patients } \\
(\mathbf{\%})\end{array}$ & $\begin{array}{l}\text { Non-diabetics } \\
(\%)\end{array}$ & $\begin{array}{l}\text { Diabetics } \\
(\%)\end{array}$ & P value \\
\hline EVD/NPS & 2 & 0.5 & 10 & 0.002 \\
\hline Wound infection & 1 & 0 & 7 & 0.004 \\
\hline CVA & 1 & 0 & 7 & 0.004 \\
\hline UTI & 2 & 1 & 10 & 0.007 \\
\hline Transfusion & 2 & 1 & 10 & 0.007 \\
\hline Pneumonia & 3 & 1 & 10 & 0.013 \\
\hline Seizure & 6 & 5 & 12 & 0.069 \\
\hline DVT & 1 & 0.5 & 2 & 0.292 \\
\hline PE & 0 & 0 & 0 & 1 \\
\hline MI & 0 & 0 & 0 & 1 \\
\hline Death & 1 & 1 & 0 & 1 \\
\hline Any complication & 12 & 8 & 32 & $<0.001$ \\
\hline
\end{tabular}

Table 2: Summary of postoperative complications following meningioma resection. Need for EVD/VPS placement and transfusion requirement in addition to rates of wound infection, CVA, UTI, and pneumonia were significantly higher in diabetics. No statistically significant difference was noted in rates of postoperative seizure, DVT, $\mathrm{PE}, \mathrm{MI}$, or death postoperatively between diabetics and non-diabetics. Overall, patients with type $2 \mathrm{DM}$ were 4.1 times more likely to experience at least one postoperative complication.

Overall, patients experienced a mean postoperative increase in KPS scores of $4.3 \pm$ 14.7. The average duration of postoperative hospitalization was $5.2 \pm 6.1$ days. Patients stayed in the ICU postoperatively for a mean duration of $3.4 \pm 4.4$ days. Two-hundred and eleven (81\%) patients had duration of follow-up of at least 90 days. Amongst this group, 54 (26\%) patients had at least one presentation to an ED within 90 days of the operation accounting for a total of 92 different presentations. Patients with type 2 DM were more likely to suffer from postoperative deterioration of performance status, have extended postoperative hospital and ICU stays, and to present to the ED with qualifying complaints within the first 3 postoperative months. These findings are summarized in Table 3.

A multiple variable logistic regression analysis adjusting for age, gender, BMI, preoperative and postoperative glucose levels, preoperative KPS, tumor grade, and extent of resection was performed to assess for an independent relationship between a pre-existing diagnosis of type $2 \mathrm{DM}$ and postoperative ED visits. Diabetic patients were found to be at higher risk of at least one postoperative $\mathrm{ED}$ presentation $(\mathrm{OR}=3.2,95 \% \mathrm{CI}=1.4$ to $7.8, \mathrm{p}=0.008)$. In multivariable linear regression analyses, a history of diabetes was predictive of worse postoperative change in KPS scores, increased durations of hospitalization, and increased length of ICU stay. These results are summarized in Table 4. 
Citation: Nayeri A, Chotai S, Douleh DG, Brinson PR, Prablek MA, et al. (2016) Type 2 Diabetes Mellitus is an Independent Risk Factor for Postoperative Complications in Patients Surgically Treated for Meningioma. J Neurol Neurophysiol 7: 368. doi: $10.4172 / 2155-9562.1000368$

Page 4 of 6

\begin{tabular}{|l|l|l|l|l|}
\hline & All patients & Non-diabetics & Diabetics & P value \\
\hline $\begin{array}{l}\text { Postoperative } \\
\text { change in KPS } \\
\text { score (mean) }\end{array}$ & 4.3 & 5.6 & -2.7 & $<0.001$ \\
\hline $\begin{array}{l}\text { Postoperative } \\
\text { decrease in KPS } \\
\text { score (\% of } \\
\text { patients) }\end{array}$ & 10 & 7 & 29 & $<0.001$ \\
\hline $\begin{array}{l}\text { Mean hospital } \\
\text { stay (days) }\end{array}$ & 5.2 & 4.7 & 7.6 & 0.004 \\
\hline $\begin{array}{l}\text { Mean ICU stay } \\
\text { (days) } 3.4\end{array}$ & 2.9 & 6 & 0.03 \\
\hline $\begin{array}{l}\text { Postoperative ED } \\
\text { presentation (\% of } \\
\text { patients) }\end{array}$ & 26 & 20 & 55 & $<0.001$ \\
\hline
\end{tabular}

Table 3: Postoperative changes in performance status, durations of hospital and ICU stay, and ED presentation after meningioma resection. Diabetics are at increased risk of postoperative deterioration, lengthier hospital and ICU stays, and present to the ED postoperatively at a higher rate than non-diabetics.

\begin{tabular}{|c|c|c|c|c|}
\hline & \multirow[t]{2}{*}{$\begin{array}{l}\text { Beta } \\
\text { coefficient }\end{array}$} & \multirow[t]{2}{*}{$P$ value } & \multicolumn{2}{|c|}{$\begin{array}{l}95 \% \quad \mathrm{Cl} \\
\text { coefficient }\end{array}$} \\
\hline & & & Upper & Lower \\
\hline \multicolumn{5}{|c|}{ Postoperative change in KPS score } \\
\hline Constant & 37.6 & $<0.001$ & 23.1 & 52.1 \\
\hline Diabetes & -7.57 & 0.003 & -12.5 & -2.68 \\
\hline Preoperative KPS & -0.32 & $<0.001$ & -0.48 & -0.17 \\
\hline Age & -0.13 & 0.034 & -0.25 & -0.1 \\
\hline \multicolumn{5}{|l|}{ Length of hospital stay } \\
\hline Constant & 13.6 & $<0.001$ & 8.53 & 18.7 \\
\hline Diabetes & 2.74 & 0.007 & 0.74 & 4.7 \\
\hline Preoperative KPS & -0.12 & 0.001 & -0.18 & -0.05 \\
\hline \multicolumn{5}{|l|}{ Length of ICU stay } \\
\hline Constant & 3.64 & 0.088 & -0.54 & 7.74 \\
\hline Diabetes & 2.1 & 0.006 & 1.1 & 4.1 \\
\hline Preoperative KPS & -0.06 & 0.017 & -0.1 & -0.01 \\
\hline $\begin{array}{l}\begin{array}{l}\text { Preoperative blood } \\
\text { glucose }\end{array} \\
\end{array}$ & 0.01 & 0.015 & 0.003 & 0.03 \\
\hline BMI & 0.74 & 0.017 & 0.013 & 0.13 \\
\hline
\end{tabular}

Table 4: Summary of the multivariable linear regression models for postoperative change in KPS scores, length of hospitalization, and duration of ICU stay. Diabetes is independently associated with postoperative deterioration in functional status in addition to lengthier hospitalization and ICU stays.

Any association between preoperative and postoperative blood glucose levels with postoperative complications, perioperative changes

in KPS score, lengths of hospitalization and ICU stay in addition to postoperative ED presentation were assessed. Elevated preoperative blood glucose levels were associated with a higher rate of postoperative complications, lengthier ICU stays, and higher risk of postoperative ED presentation $(\mathrm{p}=0.022, \mathrm{p}<0.001$, and $\mathrm{p}<0.001$ respectively). No statistically significant relationship was found between preoperative hyperglycemia and postoperative changes in KPS scores or duration of hospital stay $(\mathrm{p}=0.173$ and $\mathrm{p}=0.083$ respectively). Elevated postoperative blood glucose levels were also associated with a higher risk of postoperative complication $(\mathrm{p}=0.001)$ and $\mathrm{ED}$ presentation $(\mathrm{p}=0.020)$ but not with a postoperative decrease in KPS score $(\mathrm{p}=0.262)$ or with the length of postoperative hospital or ICU stay ( $\mathrm{p}=0.264$ and $\mathrm{p}=0.356$ respectively).

Amongst patients with diabetes, no statistically significant association was found between either preoperative or postoperative blood glucose levels and risk of postoperative complications, deterioration of performance status, or length of stay in the hospital or the ICU. Elevated postoperative blood glucose levels were found to be predictive of postoperative presentation to the ED ( $\mathrm{p}=0.041)$. No statistically significant association was found between preoperative blood glucose levels and risk of postoperative ED presentation. These results are summarized in Table 5 .

\begin{tabular}{|l|c|c|}
\hline & $\begin{array}{l}\text { Preoperative } \\
\text { blood glucose }\end{array}$ & $\begin{array}{c}\text { Postoperative } \\
\text { blood glucose }\end{array}$ \\
\hline Postoperative ED presentation & 0.067 & 0.041 \\
\hline Any postoperative complication & 0.413 & 0.053 \\
\hline Postoperative change in KPS score & 0.766 & 0.852 \\
\hline Duration of hospital stay & 0.989 & 0.862 \\
\hline Duration of ICU stay & 0.392 & 0.425 \\
\hline
\end{tabular}

Table 5: Prognostic significance of preoperative and postoperative hyperglycemia amongst diabetics after meningioma resection. Shown below are $\mathrm{p}$ values representing the association of blood glucose levels before and after the operation with postoperative complications, changes in performance status, hospitalization duration, and postoperative ED presentation. Postoperative hyperglycemia amongst diabetics is associated with an increased risk of subsequent presentation to the ED.

\section{Discussion}

In the past several years, there has been increased recognition of the elevated risk of perioperative complications, increased healthcare costs, and post-discharge readmission rates amongst diabetics undergoing a variety of different operations [11-15]. With regard to neurosurgical procedures, type $2 \mathrm{DM}$ has previously been shown to predict higher rates of certain neurological complications after craniotomies. We sought to expand on this work and assess the relative risks of a variety of neurological and non-neurological complications in addition to perioperative performance status changes, duration of postoperative hospitalization, and postoperative ED presentations in patients undergoing the resection of a primary intracranial meningioma.

Our focus on meningiomas in particular was partly motivated by the underappreciated heterogeneity of outcomes in this patient population. Despite being generally regarded as benign tumors with 
Citation: Nayeri A, Chotai S, Douleh DG, Brinson PR, Prablek MA, et al. (2016) Type 2 Diabetes Mellitus is an Independent Risk Factor for Postoperative Complications in Patients Surgically Treated for Meningioma. J Neurol Neurophysiol 7: 368. doi: $10.4172 / 2155-9562.1000368$

Page 5 of 6

low risks of perioperative morbidity and mortality, our experience with operative meningiomas has demonstrated significant postoperative complications and morbidity in select subgroups of patients. A review of the current literature on this topic only identifies a select number of non-modifiable risk factors as consistently predicting increased rates of perioperative complications following meningioma resection [6-10]. Our results indicate that a pre-existing diagnosis of type $2 \mathrm{DM}$ should be considered alongside increased patient age, poor functional capacity, and tumor location in the proper risk stratification of patients undergoing surgical resection of an intracranial meningioma. With a continued increase in the prevalence of type $2 \mathrm{DM}$, this negative prognostic relationship will pertain to a significant number of patients and should be carefully considered in the patient selection for operative resections and the perioperative management of those patients who are surgically treated [21,22].

In contrast to the other predictors of postoperative complications following meningioma resection, a diagnosis of diabetes and the associated hyperglycemia may provide an opportunity to intervene on a potentially modifiable risk factor for perioperative complications. In 2012, Davis et al. demonstrated that even slight preoperative hyperglycemia is a predictor of postoperative complication risk in addition to prolonged hospital and ICU stay following neurosurgical intervention [23]. They suggested that tighter glycemic control may be a way to reduce the burden of perioperative morbidity in diabetics undergoing neurosurgical procedures. In our study, we were able to demonstrate that both preoperative and postoperative hyperglycemia were associated with increased risks of postoperative complications and subsequent presentation to the ED. Moreover, preoperative hyperglycemia was associated with a significantly increased mean duration of ICU stay postoperatively. Our study confirms the previous findings regarding the increased risks of complicated postoperative periods in the context of poorly controlled blood glucose levels and directly connects this notion to patients undergoing a meningioma resection.

The significance of perioperative glycemic control amongst diabetics is less clear in our results. We were able to show that postoperative hyperglycemia is associated with a higher risk postoperative ED presentation amongst diabetics. However, we did not find any statistically significant association between perioperative glycemic control and postoperative complications. Certainly, this study may lack the statistical power necessary to elucidate such a relationship. With only 41 diabetics included in this study, we were able to hint at a potential association between postoperative blood glucose levels and postoperative complications $(\mathrm{p}=0.053)$. It may be that with a larger sample size of patients' further relationships between the degree of perioperative glycemic control and postoperative complications amongst diabetics may be identified. We also question the reliability of spot blood glucose measurements as an indicator of a patient's blood glucose control. Ideally, a more accurate way to assay our patients' long-term glycemic management would be via measurement of hemoglobin A1C levels. Unfortunately, hemoglobin A1C levels are not routinely collected in our practice and were not available for analysis in this study. Overall, future studies with larger cohorts of patients and better markers for long-term glycemic control are needed to better characterize the relationship between hyperglycemia and postoperative complications amongst diabetics undergoing meningioma resection.

Regardless of the role tighter glycemic control may have in reducing the rate of postoperative complications in this patient population, there are a number of steps that may be taken in an effort to better care for diabetic patients undergoing a meningioma resection. Increased vigilance and better recognition of a number of complications including a UTI, pneumonia, or a wound infection may help identify and treat these issues earlier and more effectively. Other complications, such as a cerebrovascular accident or need for a ventricular drain or shunt, may be difficult to anticipate or better treat even with increased vigilance or surveillance. However, it is key to recognize that these complications are significantly more common amongst diabetics, and this should be carefully considered before proposing an operative resection for such patients and when preoperative counseling.

Anticipated performance status changes are also vital to the appropriate patient selection and counseling for elective meningioma resection. We showed that a pre-existing diagnosis of type 2 DM portends relatively poor changes in performance status. In fact, in our study most diabetics experienced a postoperative deterioration in performance status. We believe that this prognostic relationship should play a role in the risk stratification of patients for the elective resection of an intracranial meningioma. Moreover, for diabetic patients in whom an operative resection is pursued, preoperative counseling regarding the relatively high risk of deterioration of performance status should take place.

In addition to changes in performance status and postoperative complications, we sought to assess the rates of complications following initial postoperative discharge. We selected a postoperative ED presentation within 90 days of the operation as our measure of subacute complications following meningioma resection. The significant proportion of our diabetics with such an ED presentation (48\%) raises significant concerns. In 2015, our group identified a postoperative ED presentation after meningioma resection as a predictor of tumor recurrence and increased mortality [24]. This indirect relationship may potentially be used to further risk stratify diabetics for the surgical resection of a meningioma. Moreover, a postoperative ED visit has also been previously studied as an important marker of quality of care [25-27]. Increased frequency of early postoperative follow-up may be warranted in diabetic patients undergoing a surgical resection of a meningioma in an attempt to reduce the burden of ED presentation.

Another common marker of quality of care is the duration of postoperative hospital and ICU stay. Lengthier hospitalization times are associated with decreased patient satisfaction, increased costs to the patients and the healthcare system, and predict an elevated risk of nosocomial infections and iatrogenic complications [28-30]. In our study we identified diabetes as an independent risk factor for a lengthier duration of postoperative ICU and hospital stays. In fact, we found that diabetics experienced hospitalizations and ICU stays roughly 3 days longer on average than non-diabetics. We also noted that preoperative hyperglycemia was associated with lengthier ICU stays postoperatively. However, when looking specifically at diabetics, neither preoperative nor postoperative hyperglycemia was predictive of lengthier durations of hospitalization or ICU stays. Again, our study may be underpowered to properly assess the relationship of hyperglycemia in diabetics with lengthier postoperative hospitalizations. Furthermore, long-term glycemic control as assayed by hemoglobin A1C levels may have a stronger association with postoperative complications and lengthier hospitalization. As mentioned earlier, we recommend a larger scale study, preferably with hemoglobin A1C lab collection, to better assess the relationship of hyperglycemia to postoperative complications and longer durations of hospitalization amongst diabetics. 
Citation: Nayeri A, Chotai S, Douleh DG, Brinson PR, Prablek MA, et al. (2016) Type 2 Diabetes Mellitus is an Independent Risk Factor for Postoperative Complications in Patients Surgically Treated for Meningioma. J Neurol Neurophysiol 7: 368. doi: $10.4172 / 2155-9562.1000368$

Page 6 of 6

\section{Study Limitations}

This study has a number of limitations. The design as a retrospective cohort study only allows for inference of association instead of causation. A pre-existing history of type 2 DM was defined as the clinical variable of interest and cases of diabetes subsequent to the diagnosis of meningioma were not assessed. In our practice, hemoglobin A1C levels is not routinely collected and thereby we have no measure of this cohort's long-term glycemic management prior to surgical resection. Lastly, the effects of antidiabetic medications on the rates of complications, risk of ED presentation, and changes in functional status are not assessed.

\section{Conclusions}

A pre-existing diagnosis of type $2 \mathrm{DM}$ is associated with increased risk of postoperative complications, lengthier hospital and ICU stays, deterioration of performance status, and increased rates of postoperative $\mathrm{ED}$ presentation following the resection of an intracranial meningioma. These negative prognostic relationships are independent of previously identified predictors of perioperative complications in meningiomas. This increased burden of postoperative complications may have significant implications for the proper patient selection for elective surgery, patient counseling, appropriate postoperative patient surveillance, and quality of care measures. Further studies are necessary to elucidate any role tighter glycemic control and anti-diabetic medications may have in reducing the burden of postoperative complications in diabetic patients undergoing an operative resection of a meningioma.

\section{References}

1. Ostrom QT, Gittleman H, Liao P, Rouse C, Chen Y, et al. (2014) CBTRUS statistical report: primary brain and central nervous system tumors diagnosed in the United States in 2007-2011. Neuro Oncol 4: iv1-63.

2. Wiemels J, Wrensch M, Claus EB (2010) Epidemiology and etiology of meningioma. J Neurooncol 99: 307-314.

3. Cahill KS, Claus EB (2011) Treatment and survival of patients with nonmalignant intracranial meningioma: results from the Surveillance, Epidemiology, and End Results Program of the National Cancer Institute. Clinical article. J Neurosurg 115: 259-267.

4. Kallio M, Sankila R, Hakulinen T, Jääskeläinen J (1992) Factors affecting operative and excess long-term mortality in 935 patients with intracranial meningioma. Neurosurgery 31: 2-12.

5. Nakasu S, Fukami T, Jito J, Nozaki K (2009) Recurrence and regrowth of benign meningiomas. Brain Tumor Pathol 26: 69-72.

6. Connolly ID, Cole T, Veeravagu A, Popat R, Ratliff J, et al. (2015) Craniotomy for Resection of Meningioma: An Age-Stratified Analysis of the MarketScan Longitudinal Database. World Neurosurg 84: 1864-1870.

7. Grossman R, Mukherjee D, Chang DC, Bennett R, Brem H, et al. (2011) Preoperative charlson comorbidity score predicts postoperative outcomes among older intracranial meningioma patients. World Neurosurg 75: 279-285.

8. Mukherjee S, Thakur B, Corns R, Connor S, Bhangoo R, et al. (2015) Resection of olfactory groove meningioma - a review of complications and prognostic factors. Br J Neurosurg 29: 685-692.

9. Bartek J, Sjåvik K, Förander P, Solheim O, Gulati S, et al. (2015) Predictors of severe complications in intracranial meningioma surgery: a population-based multicenter study. World Neurosurg 83: 673-678.

10. Patil CG, Veeravagu A, Lad SP, Boakye M (2010) Craniotomy for resection of meningioma in the elderly: a multicentre, prospective analysis from the National Surgical Quality Improvement Program. J Neurol Neurosurg Psychiatr 81: 502-505.
11. Toor AS, Jiang JJ, Shi LL, Koh JL (2014) Comparison of perioperative complications after total elbow arthroplasty in patients with and without diabetes. J Shoulder Elbow Surg 23: 1599-1606.

12. McAlister FA, Man J, Bistritz L, Amad H, Tandon P (2003) Diabetes and coronary artery bypass surgery: an examination of perioperative glycemic control and outcomes. Diabetes Care 26: 1518-1524.

13. Wiseman JT, Fernandes-Taylor S, Barnes ML, Saunders RS, Saha S, et al. (2015) Predictors of surgical site infection after hospital discharge in patients undergoing major vascular surgery. J Vasc Surg 62: 1023-1031.

14. Stewart RD, Campos CT, Jennings B, Lollis SS, Levitsky S, et al. (2000) Predictors of 30-day hospital readmission after coronary artery bypass. Ann Thorac Surg 70: 169-174.

15. Pollock FH, Bethea A, Samanta D, Modak A, Maurer JP, et al. (2015) Readmission within 30 days of discharge after hip fracture care. Orthopedics 38: e7-13.

16. Chen C, Zhang B, Yu S, Sun F, Ruan Q, et al. (2014) The incidence and risk factors of meningitis after major craniotomy in China: a retrospective cohort study. PLoS One 9: e101961.

17. Hutter G, von Felten S, Sailer MH, Schulz M, Mariani L (2014) Risk factors for postoperative CSF leakage after elective craniotomy and the efficacy of fleece-bound tissue sealing against dural suturing alone: a randomized controlled trial. J Neurosurg 121: 735-744.

18. Harris PA, Taylor R, Thielke R, Payne J, Gonzalez N, et al. (2009) Research electronic data capture (REDCap) - A metadata-driven methodology and workflow process for providing translational research informatics support. Journal of Biomedical Informatics 42: 377-381.

19. Microsoft, Microsoft Excel (2011) Microsoft Corporation: Redmond, Washington. p. Computer Software.

20. (2012) IBM, SPSS. IBM Corporation: Armonk, NY: IBM Corp.

21. Centers for Disease Control and Prevention (CDC) (2003) Prevalence of diabetes and impaired fasting glucose in adults--United States, 1999-2000. MMWR Morb Mortal Wkly Rep 52: 833-837.

22. Narayan KM, Boyle JP, Thompson TJ, Sorensen SW, Williamson DF (2003) Lifetime risk for diabetes mellitus in the United States. JAMA 290: 1884-1890.

23. Davis MC, Ziewacz JE, Sullivan SE, El-sayed AM (2012) Preoperative hyperglycemia and complication risk following neurosurgical intervention: A study of 918 consecutive cases. Surg Neurol Int 3: 49.

24. Nayeri A, Douleh DG, Brinson PR, Weaver KD, Thompson RC, et al. (2015) Early postoperative emergency department presentation predicts poor long-term outcomes in patients surgically treated for meningioma. J Clin Neurosci 25: 79-83.

25. Autorino R, Zargar H, Butler S, Laydner H, Kaouk JH (2015) Incidence and risk factors for 30-day readmission in patients undergoing nephrectomy procedures: a contemporary analysis of 5276 cases from the national surgical quality improvement program database. Urology 85 : 843-849.

26. Mullen MG, Lapar DJ, Daniel SK, Turrentine FE, Hanks JB, et al. (2014) Risk factors for 30-day hospital readmission after thyroidectomy and parathyroidectomy in the United States: An analysis of National Surgical Quality Improvement Program outcomes. Surgery 156: 1423-1430.

27. Tsai TC, Joynt KE, Orav EJ, Gawande AA, Jha AK (2013) Variation in surgical-readmission rates and quality of hospital care. N Engl J Med 369: 1134-1142.

28. Dasenbrock HH, Liu KX, Devine CA, Chavakula V, Smith TR, et al. (2015) Length of hospital stay after craniotomy for tumor: a National Surgical Quality Improvement Program analysis. Neurosurg Focus 39: E12.

29. Shojania KG, Duncan BW, McDonald KM, Wachter RM (2002) Safe but sound: patient safety meets evidence-based medicine. JAMA 288: 508-513.

30. Caminiti C, Meschi T, Braglia L, Diodati F, Iezzi E, et al. (2013) Reducing unnecessary hospital days to improve quality of care through physician accountability: a cluster randomised trial. BMC Health Serv Res 13: 14. 